\title{
Serum Concentration of Selenium and GPX Enzyme Activity in Iranian Children with Asthma
}

\author{
Azardokht Tabatabaei (iD) ${ }^{1}$, Mohammad Babaee (iD ${ }^{2}$, Nariman Moradi (ii) ${ }^{3}$, Mohammad Nabavi (ii) ${ }^{4}$, Saba \\ Arshi (iD ${ }^{4}$ and Soudabeh Fallah (iD ${ }^{5,{ }^{*}}$ \\ ${ }^{1}$ Research center of Pediatric Infectious Disease, Rasool Akram Hospital, Iran University of Medical Sciences, Tehran, Iran \\ ${ }^{2}$ Department of Biochemistry, Iran University of Medical Sciences, Tehran, Iran \\ ${ }^{3}$ Cellular and Molecular Research Center, Research Institute for Health Development, Kurdistan University of Medical Sciences, Sanandaj, Iran \\ ${ }^{4}$ Asthma and Allergy Research Center, Rasool Akram Hospital, Iran University of Medical Sciences, Tehran, Iran \\ ${ }^{5}$ Department of Biochemistry, Faculty of Medicine, Iran University of Medical Sciences, Tehran, Iran \\ "Corresponding author: Department of Biochemistry, Faculty of Medicine, Iran University of Medical Sciences, Hemmat High-Way, Postal Code: 14155-5983, Tehran, Iran. Email: \\ fallah.s@iums.ac.ir
}

Received 2020 March 07; Revised 2020 May 02; Accepted 2020 June 22.

\section{Abstract}

Background: Asthma is a disease with chronic airway inflammation, whereby the airway hyper-reactivity increases. Oxidative stress balance plays a critical role in asthma.

Objectives: The present study aimed to evaluate serum glutathione peroxidase (GPX) enzyme activity and selenium levels in patients with asthma.

Methods: This case-control study was conducted on 64 children aged 1 - 5 years, 32 subjects as controls, and 32 subjects with asthma who were selected randomly. The serum GPX activity and selenium levels of the studied subjects were determined spectrophotometrically.

Results: The present study showed that serum GPX activity and Selenium (Se) levels of patients with asthma were significantly lower than those of controls $(\mathrm{P}<0.001, \mathrm{P}<0.05$, respectively).

Conclusions: Reduction of serum GPX activity and Se concentration in patients could have a potential role in the pathogenesis and modulation of asthma. Micronutrient supplementation of Se may be beneficial in asthma.

Keywords: Glutathione Peroxidase, Selenium, IgG, Oxidative Stress, Asthma

\section{Background}

Asthma, a disease with chronic airway inflammation, is categorized as allergic asthma and non-allergic (intrinsic) asthma. Allergic asthma is more prevalent than nonallergic asthma. In allergic asthma, both immune regulatory and epithelial cells work together to induce different phenotypes of chronic airway inflammation. However, in non-allergic asthma, which often develops later in life, neither IgE reactivity to allergens nor any clear involvement of the adaptive immune system, such as type 2 helper $\mathrm{T}$ cells (Th2 cells) appears. Shortness of breath, chest tightness, and wheezing are the main clinical symptoms of allergic asthma (1). Animal and clinical trial studies have revealed that oxidative stress balance plays a critical role in asthma. Increased ROS production could impair the physiological conditions of cells, though physiological levels of ROS are essential for physiological conditions (2). The elevation of ROS in asthma is accompanied by an increase in lipid peroxidation products and protein carbonyl levels. In response to many pathophysiologic changes of several antioxidants, including glutathione peroxidase (GPX), superoxide dismutase (SOD), catalase, glutathione, vitamin E, and vitamin $\mathrm{C}$ are dedicated by lung and blood to counter the toxicity mediated by oxidants (3). GPX is a membrane of associated selenium-dependent antioxidant enzymes that catalyzes the degradation of organic hydroperoxides and directly reduces phospholipid hydroperoxides and protects against oxidative stress in mammalian cells (4). Selenium (Se) is an essential component of GPX enzyme synthesis and function. Different physiological levels of Se for different populations have been attributed to its concentration in soil, vegetable, and herbivore meat (5). It is a cofactor required to maintain the activity of glutathione peroxidase, an enzyme that catalyzes the degradation of organic hydroperoxides. In asthma, a low concentration of selenium could affect the inflammatory process 
by decreasing the GPX activity, which could result in diminished protection against oxidative damage. Selenium levels might have a significant role in asthma pathogenesis. Low selenium levels of the New Zealand population reflected a low consumption of selenium (6).

\section{Objectives}

The present study aimed to investigate the serum concentration of selenium and serum GPX activity of patients with asthma disease and controls.

\section{Methods}

\subsection{Studied Subjects}

This case-control study was conducted on 64 children aged 1 - 5 years, including 32 subjects as controls and 32 IgE-positive subjects with asthma who were selected randomly, and attended at the Immunology Center of Rasoul Akram Hospital (Tehran, Iran). Asthmatic patients were screened according to guidelines given by a specialist on duty, and diagnosis of asthma was established based on measurement of IgE levels using ELISA method (data not shown), skin prick testing with common allergens, chest $\mathrm{X}$-ray, spirometry, as well as individual and family history.

The studied groups with informed consent completed a questionnaire, which contained medical history, smoking, diet, and alcohol history. Subjects with any inflammatory, cardiovascular, and malignant diseases were excluded from the study. The recipients of any medication and treatment, including selenium supplements, antioxidants, and glucocorticoids during at least 6-8 weeks (except asthmatic treatment drugs) and subjects with diabetes, renal and hepatic diseases were excluded from our study. The controls were characterized by a negative response to the mentioned questions. The study was approved by the Research Ethics Committee of the Hospital (IR.com iums.com 1395.com 28896). Subjects with positive IgE were considered patients with asthma.

\subsection{Sampling}

Blood samples of the studied subjects were collected after overnight fasting of 12 - 14 hours into $5 \mathrm{ml}$ vacutainer tubes containing anticoagulant EDTA with the serum separated, aliquoted, and stored at $-20^{\circ} \mathrm{C}$.

\subsection{Glutathione Peroxidase Enzyme Activity Assessment}

The GPx activity was determined spectrophotometrically in triplicate, using a coupled assay using glutathione peroxidase activity assay commercial kit (Abcam, USA,
Cat\#ab102530) according to its protocol. Based on a colorimetric method with a minimum detection limit of 0.5 $\mathrm{Mu} / \mathrm{mL}$, initially, $50 \mu \mathrm{L}$ glutathione reductase and $50 \mu \mathrm{L}$ glutathione were poured in 96 wells of the plate containing $50 \mu \mathrm{L}$ serum and incubated at $37^{\circ} \mathrm{C}$, for 10 minutes. Then, substrates of $50 \mu \mathrm{L}$ NADPH and $50 \mu \mathrm{L}$ Butylhydroperoxide were added for the initiation of the reaction. After incubation at $37^{\circ} \mathrm{C}$ for $10 \mathrm{~min}$, the kinetic changes of absorbance (decrease) at $340 \mathrm{~nm}$ were followed at $4 \mathrm{~min}$ The GPX activity (U/L) was determined using a standard curve.

\subsection{Selenium and IgE Determination}

Selenium levels $(\mu \mathrm{g} / \mathrm{mL})$ were determined by a flame atomic absorption spectrophotometer in triplicate, using a standard cure. Instrument: Agilent 200 AA series. Calibration: using standard solution Chemlab company $(1000 \mu / \mathrm{mL}), 4$-point calibration. QC: [Accuracy: Proficiency test (Inter laboratory), Precision: Pooled serum 2 levels]. Immunoglobulin E (IgE) was estimated through ELISA (ng/mL, Abcam Company, ab195216 No. Intra-assay; CV\%: 4.04\%, Inter-assay; CV\%: 3.53\%).

\subsection{Statistical Analysis of Data}

Paired samples $t$-test (Wilcoxon in non-parametric distribution) was used to assess the enzyme activity and selenium levels. To compare the changes between groups, according to the Kolmogorov-Smirnov test, we applied the independent-samples $t$-test (Mann-Whitney U test in case of non-parametric distribution). Linear regression and chisquare analysis were used for analyzing the correlation between variables. We analyzed all data with SPSS software version 18 (SPSS, Inc., Chicago, IL, USA). P < 0.05 was considered significant. All values were reported as mean \pm SD.

\section{Results}

As shown in Figure 1A, the GPX activity of patients was significantly lower than that of controls $(\mathrm{P}=0.001)$. The selenium level of patients was also significantly lower than that of controls $(\mathrm{P}<0.05)$ (Figure $1 \mathrm{~B})$. The age difference between patients and controls was significant $(\mathrm{P}<0.001)$ (Figure $1 C$ ). Linear regression analysis (Figure 2 ) and Pearson correlation coefficient (Table 1) showed no significant correlation between age and Se levels, age, and GPX activity of the patients with asthma.

Although there was a significant difference between the age of controls and patients, due to lack of association between age and every Se level and serum enzyme activity of the patients, the effect of age as a possible confounding factor in results has been removed. 
A

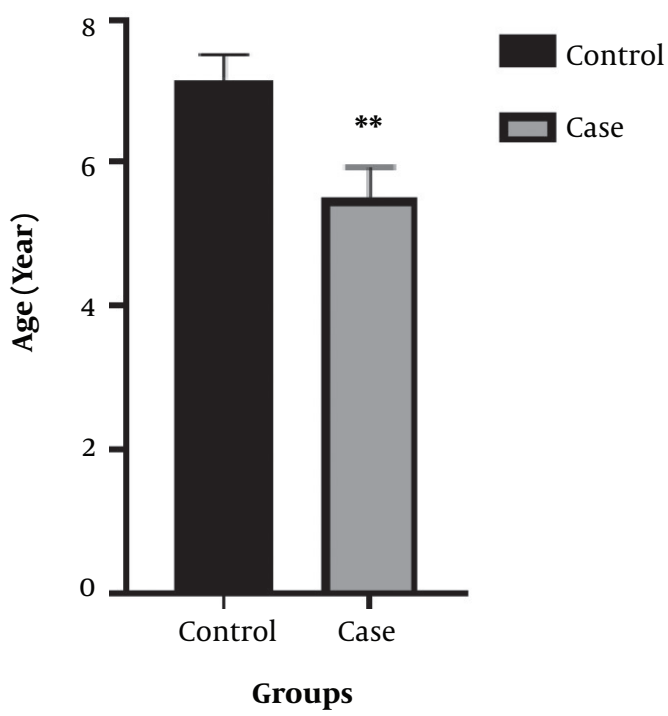

B

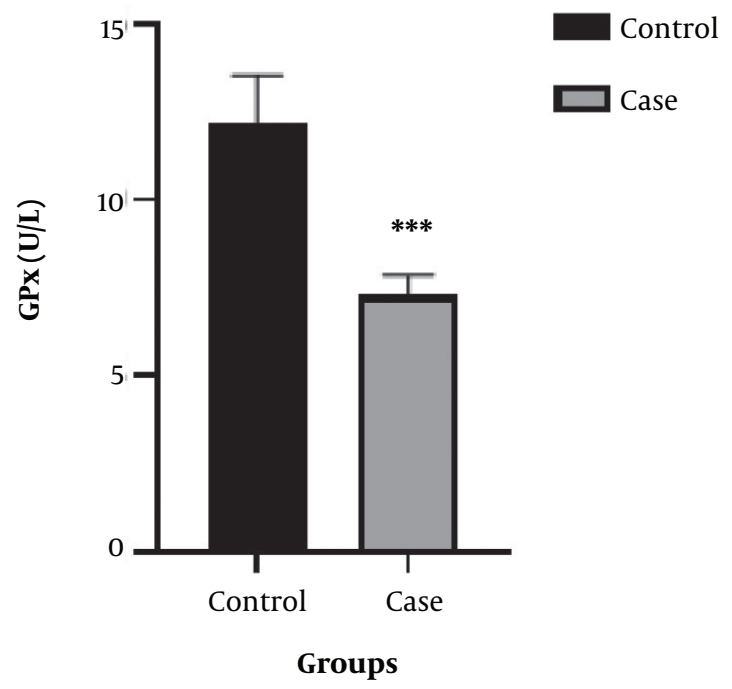

C

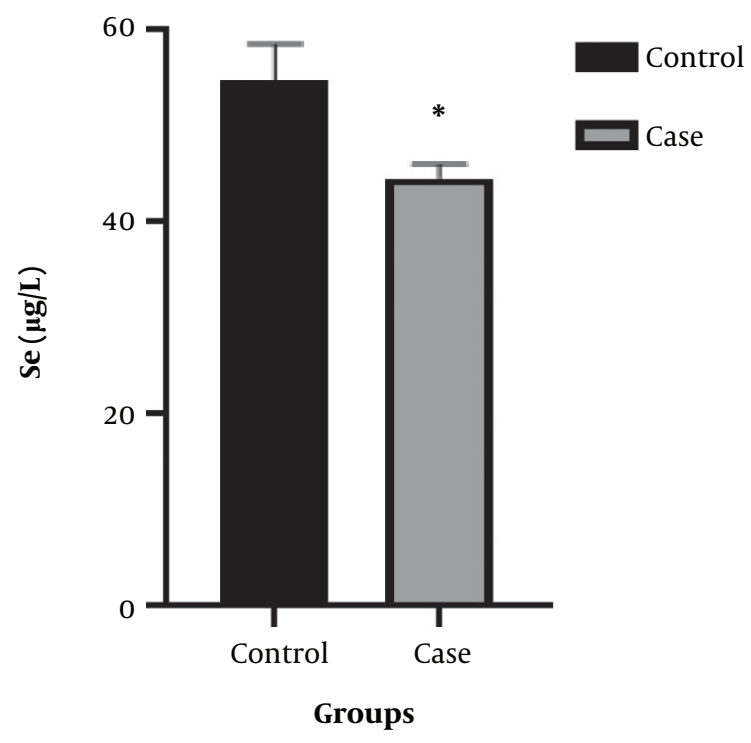

Figure 1. Comparison of the age (A), Se levels (B) and serum GPX enzyme activity (C) between studied groups. Case $=$ patients with asthma disease. ${ }^{*} \mathrm{P}<0.05,{ }^{* *} \mathrm{P}<0.01,{ }^{* * *} \mathrm{P}<$ 0.001 .

The impact of gender on serum Se concentration and GPX enzyme activity was investigated using linear regression analysis (Figure 3) and Eta coefficient calculation (Table 2) analysis. As shown in Figure 3 and Table 2, the gender had no significant effect on the correlation between serum Se concentration and serum GPX activity of the stud- ied groups.

\section{Discussion}

Antioxidants Se and GPX deficiency has been widely implicated in a variety of human diseases and their 

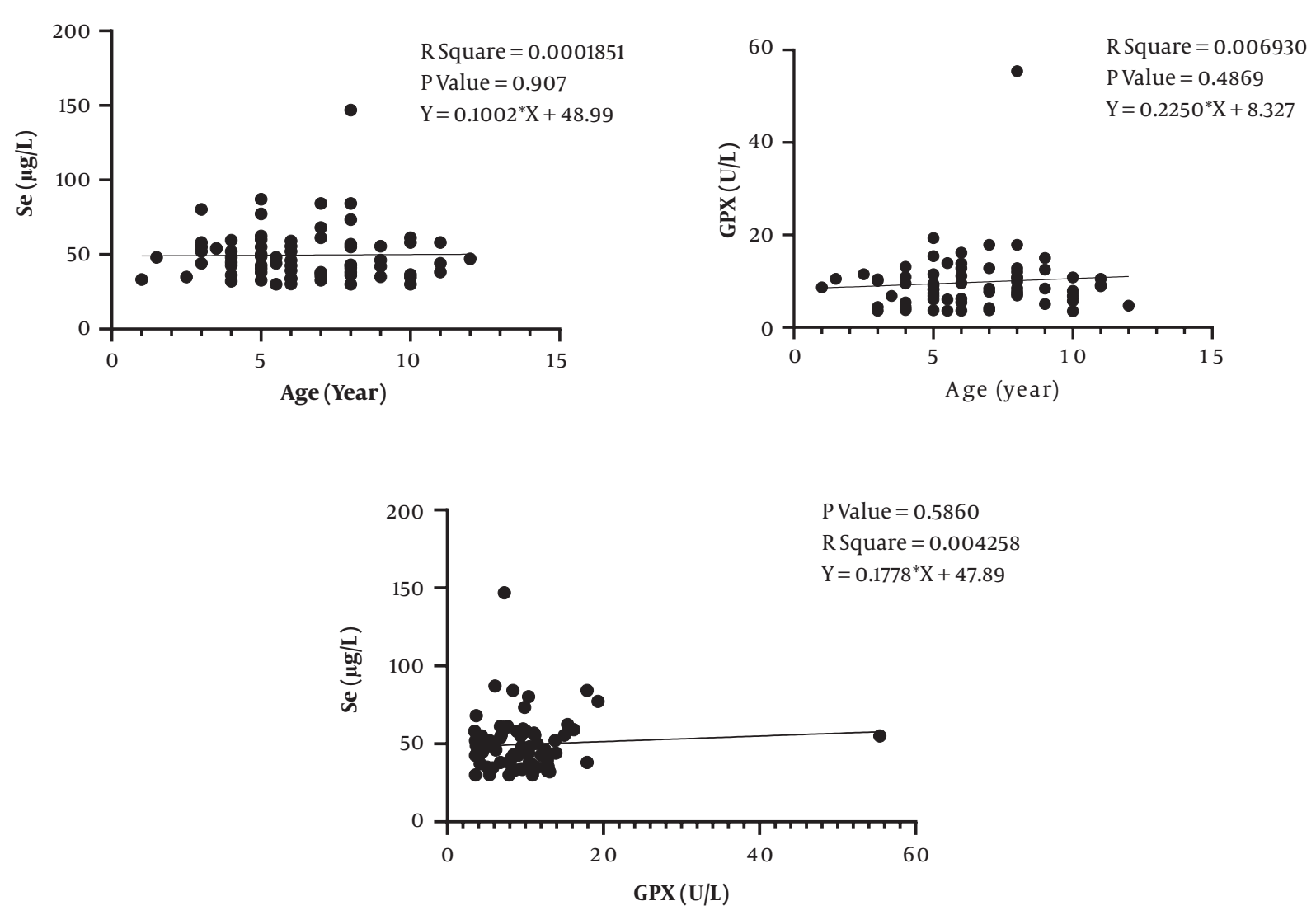

Figure 2. The linear regression of correlation between serum concentration of Se and age, serum GPX activity and age, and serum concentration of Se and GPX activity of the patients with asthma.

causes. Their levels have remained unclear in Iranian children with asthma. This study provides a comprehensive overview of two antioxidants GPX activity and Se levels of 64 children aged 1 - 5 years, 32 subjects as controls and 32 subjects with asthma disease.

The present study identified a significant reduction in serum glutathione peroxidase (GPX) activity and selenium (Se) concentration of asthmatic children as compared with controls. Se has a remarkable role in maintaining redox homeostasis, which is essential for immune system function (7). Compared to our results, a study on 27 females and 14 males, with the mean age 37.3 years with asthma, conducted by Misso et al., revealed that serum Se concentration was significantly lower than controls. They also showed that platelet GPX activity of asthmatic patients (No whole blood) was significantly lower than in nonasthmatic subjects. The reduced activity of this enzyme may be associated with the pathogenesis and severity of asthma (8). A study on 38 patients with bronchial asthma and 23 control subjects, by Nadeem et al., revealed a significant increase in oxidative stress and reduction of antiox- idant levels. Interventions increasing therapeutic antioxidant defenses could be beneficial in asthma (3). Our results are consistent with the results of a study by Amber et al., who revealed that blood selenium concentration and GSH-PX activity of 56 asthmatic patients in New Zealand were significantly lower than those of controls. Six-fold and 1.9-fold increased risk of asthma incidence was found in patients with low whole blood glutathione peroxidase activity and serum selenium concentration, respectively as compared with controls.

On the other hand, no significant decrease was found for plasma levels of selenium and GHS-PX activity of patients. The decreased serum concentration of selenium and GSH-PX activity in patients with asthma might be a result of increased oxidative stress (6). Nadeem et al. revealed an elevated concentration of serum nitrates, protein carbonyls, and lipid peroxidation products, serum sulfhydryl proteins decrease, and an increased concentration of superoxide in leukocytes of asthmatic patients studied (3). Asthma susceptibility with a number of antioxidant defense enzymes (ADE) genesis gender-dependent. 

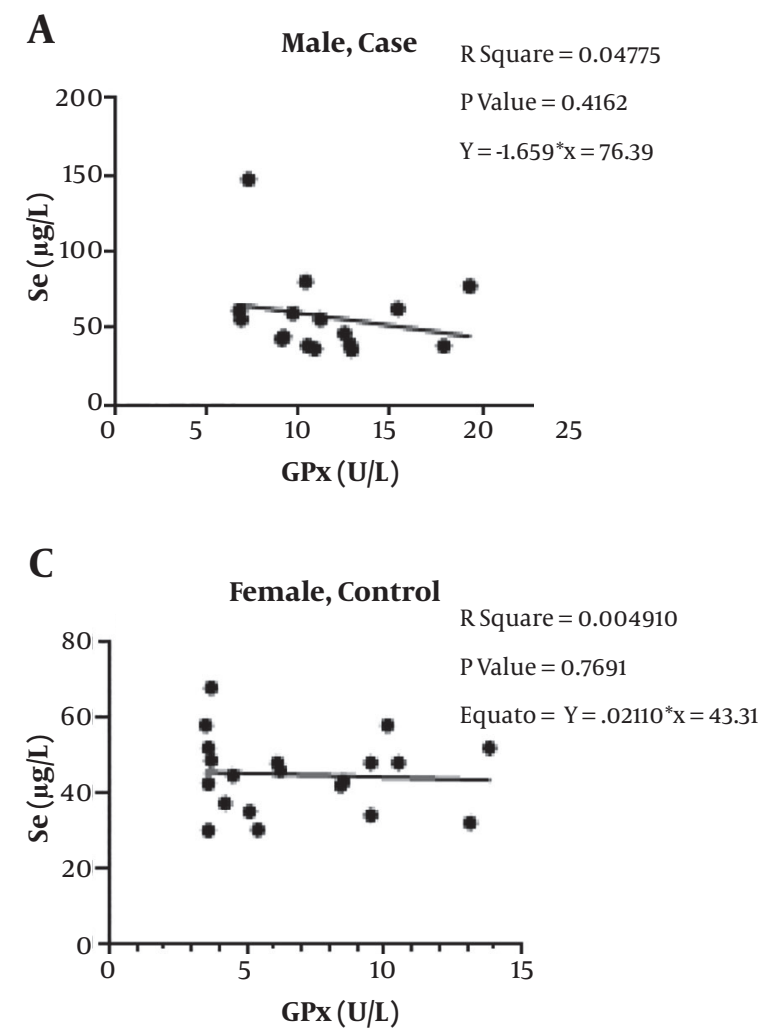

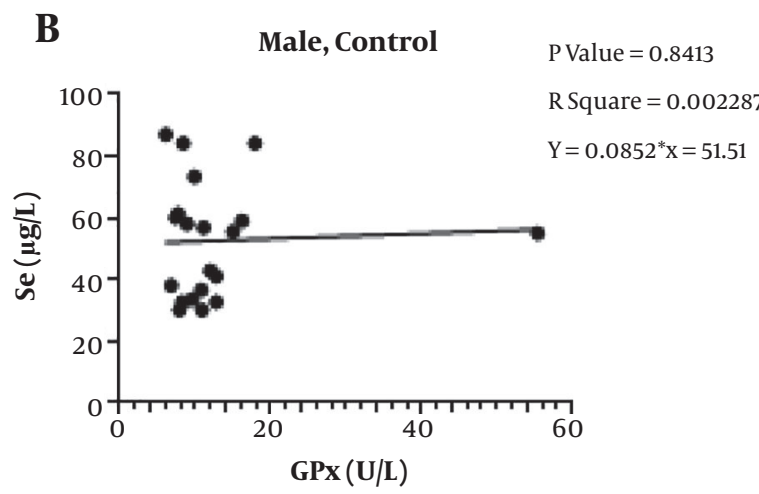

D

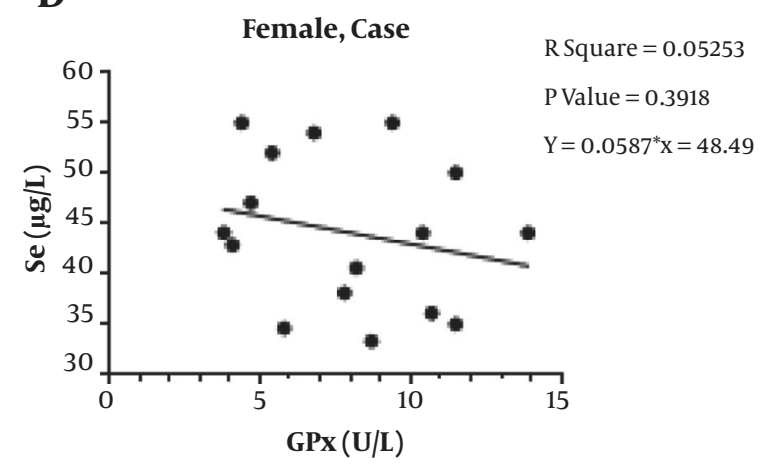

Figure 3. The linear regression of analysis of sex impact on the correlation between serum Se concentration and serum GPX activity in male and female patients with asthma. $\mathrm{GPX}=$ glutathione peroxidase enzyme; $\mathrm{Se}=$ selenium.

The gender-specific effects of ADE genes were observed on the risk of asthma. It was demonstrated that predisposition to allergic and no allergic asthma depends on discrepancy and complications of the interplay between genes involved in oxidative stress (9). It has been found that NF- $\kappa \mathrm{B}$, mitogen-activated protein kinase (MAPK), activator protein-1, and other transcription factors, will be activated by ROS/RNS (Reactive nitrogen species) causing lung inflammation (10). Incorporation of Se in GPX enzyme causes $\mathrm{H}_{2} \mathrm{O}_{2}$ reduction and prevents cell membranes from lipid peroxidation and following instability $(11,12)$. Se consumption along with other supplements, including vitamin $\mathrm{C}$, and choline chloride could reduce aryl hydrocarbon receptor (AHR), inflammation, and oxidative stress through inducing the interleukin-10 (IL-10) expression via FoxP3 (+) signaling pathway (13). Prescribing organic Se (Pro Se) without accumulation in liver and kidney and with lower toxicity as compared with inorganic Se, to asthma patients could increase the endogenous antioxidant enzyme levels (14). It has been shown that giving back and repairing of GSH and SOD activities in asthma patients plays an important role in reducing airway inflammation and remodel- ing through anti-oxidative stress $(15,16)$.

Contrary to our results, the results of a pooled-analysis by Mao et al. on 2,108 asthma patients and 8,479 controls, including eleven studies performed on Caucasians, five on Asians and one on Africans., showed no marked difference between Se levels of asthma patients and controls among the overall populations, Caucasians, Asians, and Africans (17). Consistent with our result, the result of a metaanalysis study on twenty-six studies for zinc (Zn) and forty studies for Se of relevant asthma articles in PubMed, Web of Science, and Scopus databases up to May 2019 showed that patients with asthma had low Se and Zn levels compared with healthy controls (18).

A birth cohort study was conducted by Wei Choo et al. on 132 newborn infants who were followed up for several childhood atopic diseases, including eczema, allergic rhinitis, and asthma. Atopic diseases eczema, allergic rhinitis, and asthma alone were predominantly diagnosed in 9, 59, and 10 children, respectively, at 7 years of age. They revealed that a decrease in the activity of GPX and myeloperoxidase (MPO) levels correlated with an increase in allergen-specific IgE levels. They also showed that 
Table 1. Pearson Correlation Coefficient Between Serum GPX Activity, Se Levels, and Age of Studied Groups

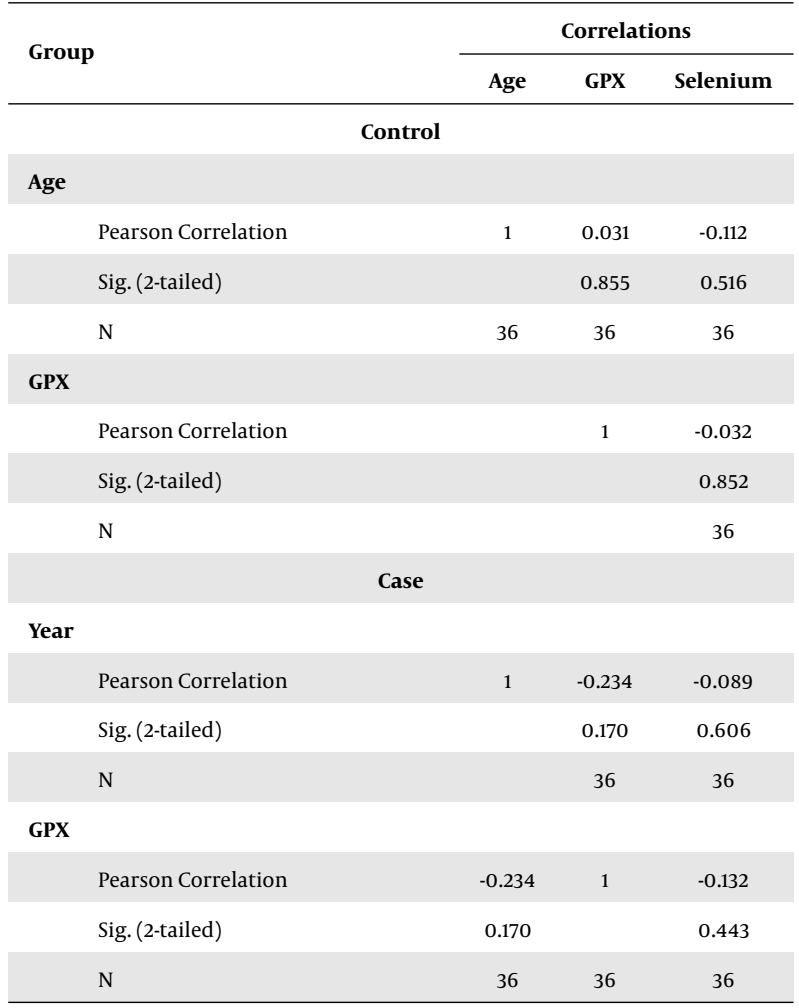

a decline in urinary 8-OHdG levels correlated with an increase in FeNO levels. Total IgE levels and the prevalence of sensitization to house dust mites were significantly higher in children with atopic diseases compared to the healthy controls. It was concluded that decreased TAC levels correlated with reduced pulmonary function tests. Oxidative stress potentially plays a role in the modulation of allergic responses contributing to atopic diseases (19). Asthma is associated with an increased oxidant burden and a decreased antioxidant defense in the systemic circulation and locally in the airway. A study by Celik et al. on 28 children aged 6 - 18 years with asthma, 17 with allergic rhinitis, 100 asthma and allergic rhinitis, and 74 healthy controls showed lower malondialdehyde levels and higher GSH levels in both nasal and oral exhaled breath condensates (EBC) samples of all patient groups compared to healthy controls. There was no difference between the different patient groups (18). A study on Nigerian children with asthma showed low levels of $\mathrm{Zn}$, Se, and total antioxidants compared to controls. Also, Se levels of patients with moderate/severe disease were lower than those of patients with mild disease (20). The effect of the Mediterranean diet on various outcomes of asthma (wheeze) and severity in children was investigated in a systematic review. The results showed that the Mediterranean diet could decrease the incidence of the flowing wheeze, flowing severe wheeze, and asthma ever. A systematic review revealed that vitamin A intake in patients with asthma was lower than in controls. The risk of asthma occurrence in patients with the lowest vitamin $C$ was $12 \%$ higher than in other patients. Although vitamin E intake was found to be unrelated to asthma, in patients with severe asthma, it was significantly lower than in mild asthma. Many studies reported a correlation between maternal antioxidant status and asthma onset during childhood. Despite some epidemiological studies in which patients with low dietary intake of antioxidants, including Se, as well as antioxidant vitamins C, E, had usually more symptoms of the disease, so far no compound has proven to be clinically useful in the modulation or recovery of the disease (21).

\subsection{Conclusion}

These findings suggest that aberrant decrease in serum GSH-PX activity and selenium concentration could have a potential role in the pathogenesis and modulation of asthma and other allergic disorders in Iranian children with asthma. Micronutrient supplementation such as Se might be beneficial in Iranian children with asthma as they have relatively lower antioxidants Se and GPX than controls.

\subsection{Study Limitations}

In this study, the small sample size was investigated, and these observations should be confirmed in a larger sample of patients with further analysis. The impact of social and economic conditions of studied groups was not negligible. We analyzed only two independent variables; other probable variables involved in the incidence of asthma should be considered in future studies.

\section{Acknowledgments}

This work was supported by a grant (Grant number 229551) from Iran University of Medical Sciences.

\section{Footnotes}

Authors' Contribution: Conceptualization: Azardokht Tabatabaei, Soudabeh Fallah. Formal analysis: Mohammad Babaee. Funding acquisition: Azardokht Tabatabaei. Investigation: Mohammad Saba Arshi. Methodology: Azardokht Tabatabaei, Soudabeh Fallah.

Conflict of Interests: No conflict of interest to declare.

Ethical Approval: The study was approved by the Research Ethics Committee of the Hospital (IR.com iums.com 


\begin{tabular}{|c|c|c|c|c|}
\hline \multirow{2}{*}{ Group } & \multicolumn{4}{|c|}{ Directional Measures } \\
\hline & & & & P Value \\
\hline Control & Nominal by Interval & Eta & GPX Dependent & 0.085 \\
\hline Patient & Nominal by Interval & Eta & GPX Dependent & 0.173 \\
\hline Control & Nominal by Interval & Eta & Selenium Dependent & 0.106 \\
\hline Patients & Nominal by Interval & Eta & Selenium Dependent & 0.046 \\
\hline
\end{tabular}

1395.com 28896). Subjects with positive IgE were considered patients with asthma.

Funding/Support: This work was supported by a grant (Grant number 229551) from Iran University of Medical Sciences.

\section{References}

1. Qu J, Li Y, Zhong W, Gao P, Hu C. Recent developments in the role of reactive oxygen species in allergic asthma. J Thorac Dis. 2017;9(1):E3243. doi: 10.21037/jtd.2017.01.05. [PubMed: 28203435]. [PubMed Central: PMC5303105].

2. Zuo L, Ni L, Chuang C. Allergic Asthma Pathogenesis and Antioxidant Therapy. Frontiers in Clinical Drug Research-Anti Allergy Agents. 2016:4579. doi: $10.2174 / 9781681081595116020004$

3. Nadeem A, Chhabra SK, Masood A, Raj HG. Increased oxidative stress and altered levels of antioxidants in asthma. J Allergy Clin Immunol. 2003;111(1):72-8. doi: 10.1067/mai.2003.17. [PubMed: 12532099].

4. Cowan DC, Taylor DR, Peterson LE, Cowan JO, Palmay R, Williamson A, et al. Biomarker-based asthma phenotypes of corticosteroid response. J Allergy Clin Immunol. 2015;135(4):877-83 e1. doi: 10.1016/j.jaci.2014.10.026. [PubMed: 25488689]. [PubMed Central: PMC4388771].

5. Fenech AG, Ellul-Micallef R. Selenium, glutathione peroxidase and superoxide dismutase in maltese asthmatic patients: effect of glucocorticoid administration. Pulm Pharmacol Ther. 1998;11(4):301-8. doi: 10.1006/pupt.1998.0122. [PubMed: 10101748].

6. Flatt A, Pearce N, Thomson CD, Sears MR, Robinson MF, Beasley R. Reduced selenium in asthmatic subjects in New Zealand. Thorax. 1990;45(2):95-9. doi: 10.1136/thx.45.2.95. [PubMed: 2315881]. [PubMed Central: PMC462313].

7. Guo CH, Liu PJ, Hsia S, Chuang CJ, Chen PC. Role of certain trace minerals in oxidative stress, inflammation, CD4/CD8 lymphocyte ratios and lung function in asthmatic patients. Ann Clin Biochem. 2011;48(Pt 4):344-51. doi: 10.1258/acb.2011.010266. [PubMed: 21546427].

8. Misso NLA, Powers KA, Gillon RL, Stewart GA, Thompson PJ. Reduced platelet glutathione peroxidase activity and serum selenium concentration in atopic asthmatic patients. Clinical Experimental Allergy. 1996;26(7):838-47. doi: 10.1046/j.1365-2222.1996.d01-376.x.

9. Polonikov AV, Ivanov VP, Bogomazov AD, Freidin MB, Illig T, Solodilova MA. Antioxidant defense enzyme genes and asthma susceptibility: gender-specific effects and heterogeneity in gene-gene interactions between pathogenetic variants of the disease. Biomed Res Int. 2014;2014:708903. doi: 10.1155/2014/708903. [PubMed: 24895604]. [PubMed Central: PMC4026955].
10. Zhu L, Ni Z, Luo X, Wang X. Advance of antioxidants in asthma treatment. World Journal of Respirology. 2017;7(1):17. doi: 10.5320/wjr.v7.i1.17.

11. Bray TM, Bettger WJ. The physiological role of zinc as an antioxidant. Free Radical Biology and Medicine. 1990;8(3):281-91. doi: 10.1016/08915849(90)90076-u.

12. Machlin LJ, Bendich A. Free radical tissue damage: protective role of antioxidant nutrients 1. The FASEB Journal. 1987;1(6):441-5. doi: 10.1096/fasebj.1.6.3315807.

13. Bansal P, Saw S, Govindaraj D, Arora N. Intranasal administration of a combination of choline chloride, vitamin C, and selenium attenuates the allergic effect in a mouse model of airway disease. Free Radic Biol Med. 2014;73:358-65. doi: 10.1016/j.freeradbiomed.2014.05.018. [PubMed: 24905385].

14. Min-Chang G, Wei-Hong T, Zhen X, Jie S. Effects of SeleniumEnriched Protein from Ganoderma lucidum on the Levels of IL-1 beta and TNF- alpha , Oxidative Stress, and NF- kappa B Activation in Ovalbumin-Induced Asthmatic Mice. Evid Based Complement Alternat Med. 2014;2014:182817. doi: 10.1155/2014/182817. [PubMed: 24660015]. [PubMed Central: PMC3934624].

15. Nader MA. Inhibition of airway inflammation and remodeling by sitagliptin in murine chronic asthma. Int Immunopharmacol. 2015;29(2):761-9. doi: 10.1016/j.intimp.2015.08.043. [PubMed: 26362207].

16. Abdel-Fattah MM, Messiha BA, Salama AA. Assessment of the Mechanistic Role of Cinnarizine in Modulating Experimentally-Induced Bronchial Asthma in Rats. Pharmacology. 2015;96(3-4):167-74. doi: 10.1159/000438705. [PubMed: 26304475].

17. Mao S, Wu L, Shi W. Prevalence and distribution patterns of allergens among children with asthma and asthma-like symptoms in Shanghai, China. Respir Res. 2020;21(1):57. doi: 10.1186/s12931-020-13181. [PubMed: 32070330]. [PubMed Central: PMC7029476].

18. Celik M, Tuncer A, Soyer OU, Sackesen C, Tanju Besler H, Kalayci O. Oxidative stress in the airways of children with asthma and allergic rhinitis. Pediatr Allergy Immunol. 2012;23(6):556-61. doi: 10.1111/j.13993038.2012.01294.x. [PubMed: 22435922].

19. Wei Choo CY, Yeh KW, Huang JL, Su KW, Tsai MH, Hua MC, et al. Oxidative stress is associated with atopic indices in relation to childhood rhinitis and asthma. J Microbiol Immunol Infect. 2020. doi: 10.1016/j.jmii.2020.01.009. [PubMed: 32094074].

20. Kuti BP, Kuti DK, Smith OS. Serum Zinc, Selenium and Total Antioxidant Contents of Nigerian Children with Asthma: Association with Disease Severity and Symptoms Control.J Trop Pediatr. 2019. doi: 10.1093/tropej/fmz078. [PubMed: 31747014].

21. Sahiner UM, Birben E, Erzurum S, Sackesen C, Kalayci O. Oxidative stress in asthma: Part of the puzzle. Pediatr Allergy Immunol. 2018;29(8):789-800. doi: 10.1111/pai.12965. [PubMed: 30069955]. 